\title{
Intersection-Connectivity-Based Roadside-Unit Allocation
}

\author{
Soyoung Park ${ }^{*}$ and Jeonghee Chi \\ Division of Internet and Multimedia Engineering, Konkuk University \\ \{soyoungpark, jhchi\}@konkuk.ac.kr
}

\begin{abstract}
The efficient and economical deployment of roadside units for the guaranteeing of stable and seamless inter-vehicle and vehicle-to-infrastructure communications is one of the most essential issues for the implementation of a practical vehicular ad-hoc networking environment. In this paper, the concept of intersection connectivity, which represents the traffic-message delivery probability between any two intersections, is completely formalized. Then, novel roadside-unit allocation strategies are proposed based on the new intersection connectivity, which can maximize both the collection and the dissemination of traffic messages by roadside units. To achieve the authors' goal, affinity propagation is exploited to cluster the intersections based on the new intersection connectivity. Notably, most roadways are bidirectional, and the connectivity between two intersections is different from the road direction; therefore, the best positions for the maximizing of traffic-data collection could be different from the best positions for the maximizing of traffic-data dissemination. Dedicated roadside-unit allocation algorithms are therefore proposed to find the optimal roadside-unit candidates for the maximizing of both message collection and dissemination. The simulated performance of the proposed scheme is analyzed using the real-field traffic data of the Seochogu district of Seoul, Korea, and the superior performance of the proposed model is shown through a comparison with the existing clustering-based approaches
\end{abstract}

Keywords: RSU allocation, Intersection connectivity, Affinity Propagation

\section{Introduction}

In a vehicular ad-hoc networking (VANET) environment, roadside units that act as traffic-message transmitters are essential infrastructural units that support seamless networking for vehicle-to-vehicle and vehicle-to-infrastructural-unit communications. Since roadside units (RSU) are usually supposed to be statically installed on the roads, RSU positions cannot be changed dynamically or frequently according to the traffic situation; therefore, the determination of the initial deployment of the RSUs is the mostimportant process of the RSU allocation in a VANET. So far, many RSU-allocation strategies for various purposes have been proposed.

The authors' goal is a deployment of RSUs that maximizes the RSU coverage for intersections, and also maximizes both the traffic-message collection and dissemination by the RSUs. An RSU covers an intersection if, and only if, the traffic-message delivery between the RSU and the intersection can be guaranteed. Here, to measure the RSU coverage for intersections, it should be possible to measure the message-delivery possibility between the RSUs and the intersections. Since RSUs are supposed to be installed at intersections, the message-delivery possibility between intersections should be defined first. In the VANET environment, the message-delivery rate is mainly determined by the amount of vehicles moving through the intersections and the distance between the intersections. The distance between the intersections is fixed, but the traffic volume

* Corresponding Author 
passing through the intersections changes dynamically according to the traffic situation, locations, and time slot. Nevertheless, a tendency of the traffic flows between intersections can be discovered based on the accumulated traffic statistics for every intersection. This tendency has been constructed according to the locational particularity (e.g. habitual congestion zone, town center, main street, commercial district, residential district, shopping-mall area, etc.) of the intersections, so it does not change frequently. With this knowledge, a probabilistic traffic-message-delivery model is defined in this paper by considering the traffic flows between intersections.

A concept of intersection connectivity that represents the message-delivery probability between a pair of intersections is introduced here, and some related RSU-allocation algorithms have been proposed as preliminary results [2-5]. In this paper, the concept of intersection connectivity for two arbitrary intersections is redeemed and finally formalized, and a new RSU-allocation strategy is then proposed based on the new intersection connectivity.

The proposed strategy creates clusters of intersections whereby the intersections have relatively higher intersection connectivity to the head intersection of a cluster compared with that regarding the other cluster heads; that is, the traffic messages that are collected at intersections in a cluster will be delivered to the head intersection of the cluster with a relatively higher probability. The cluster heads can therefore become RSU candidates for traffic-message collection. An affinity propagation (AP) algorithm [6] is exploited for the clustering of the intersections. Likewise, clusters of the intersection are found for the message dissemination; that is, the message collected at the cluster head will be delivered to the intersections in the cluster with a relatively higher probability. Most roadways, however, are bidirectional. For two arbitrary intersections denoted as $I_{A}$ and $I_{B}$, the connectivity from $I_{A}$ to $I_{B}$ can be different from the connectivity from $I_{B}$ to $I_{A}$. The best positions for traffic-data collection will consequently be different than the best positions for traffic-data dissemination; therefore, dedicated RSU-allocation algorithms are proposed to find the optimal RSU-candidate intersections for the maximizing of both message collection and dissemination.

The performance of the proposed RSU-allocation model is simulated using the realfield traffic data from Seochogu in Seoul, Korea. The simulated performance of the proposed model is analyzed for the minimum number of RSUs, the average message collection, and the dissemination connectivity through a comparison with the authors' previous works.

The related works are briefly reviewed in Section 2, and the intersection connectivity is formalized in Section 3. In Section 4, the detailed algorithms of the proposed RSUallocation scheme are provided, and the simulated performance is analyzed in Section 5. The conclusion of the paper is given in Section 6.

\section{Related Works}

Recently, many diverse RSU-allocation strategies have been proposed. The trafficdensity-based approach [1] deploys RSUs in the areas where the density of vehicles is relatively sparse to increase the vehicle connectivity. The network-based approach deploys RSUs to maximize the contacting probability with the RSUs. M. Rios et al. [9] suggested an RSU-deployment strategy that allows any public bus to communicate with an RSU within two hops in a public bus-transport network. The partition-based approach deploys RSUs to maximize the number of vehicles making contact with RSUs. Patil and Gokhale [8] proposed Voronoi-based RSU-deployment algorithms. C. M. Silva et al. [7] partitioned roads into grid cells, and then found RSU locations using both the traffic volume in a grid cell and the migration ratios between adjacent cells.

The concept of intersection connectivity was introduced by the authors for the first time in [2], and intersection connectivity-based RSU-allocation strategies were proposed 
using a Markov clustering algorithm in [4] and [5]. The goal of the authors' previous works is the maximization of the message-delivery probability to RSUs. But RSU message dissemination was not considered. Wang et al. [10] proposed an RSU-allocation scheme using an AP algorithm, for which the traffic influence between intersections was considered. The traffic influence involves a consideration of the traffic volume, distance, and speed, but it ignores the moving trajectories of vehicles between intersections; therefore, it does not reflect the traffic flow between intersections accurately. The authors' intersection connectivity is a probabilistic traffic model for which the moving trajectories of vehicles between intersections are considered, and the proposed model deploys RSUs to maximize both message collection and dissemination.

\section{Intersection Connectivity}

The concept of intersection connectivity is formalized in this paper concretely. The intersection connectivity for two adjacent intersections is firstly defined, and then, it is generalized for any two intersections. Suppose that two adjacent intersections are denoted as $I_{i}$ and $I_{j}$. The intersection connectivity from $I_{i}$ to $I_{j}$, denoted as $C_{i, j}$, is the probability that the traffic information obtained at the intersection $I_{i}$ can be delivered to the intersection $I_{j}$. Let the Euclidean distance between $I_{i}$ and $I_{j}$ be $D_{i, j}$. The traffic data generated by the vehicles located at intersection $I_{i}$ can be delivered directly to the vehicles at intersection $I_{j}$ through wireless communication if $D_{i, j}$ is within the wirelesstransmission range that is denoted as $\delta$. The traffic-message delivery can be always guaranteed in this case.

In a case where $D_{i, j}$ is greater than $\delta$, the traffic messages can be delivered by vehicles passing through both the intersections $I_{i}$ and $I_{j}$. If there are enough vehicles on the roadways between $I_{i}$ and $I_{j}$, then the traffic messages can be delivered by hop-by-hop propagation by those vehicles on the roadway. If there is no intermediate vehicle for the message propagation on the roadway, the traffic messages can be delivered to $I_{j}$ by vehicles moving from $I_{i}$ to $I_{j}$ in the manner of carry-and-forward. Consequently, in both situations, the traffic-message delivery depends on the vehicles passing through both the intersections $I_{i}$ and $I_{j}$; therefore, in the latter case, the traffic-message delivery probability is determined by the probability of encountering vehicles passing by both of the two adjacent intersections. So when a vehicle $V_{A}$ (that is not supposed to head for $I_{j}$ ) has arrived at the intersection $I_{i}$, if there is any vehicle $V_{B}$ moving toward $I_{j}$ within the transmission range of $V_{A}$ during a given time, then the traffic message obtained from $V_{A}$ can be delivered to $I_{j}$ by $V_{B}$. The traffic-message delivery probability is therefore the same as the probability that there is at least one vehicle heading for $I_{j}$ when a vehicle has arrived at $I_{i}$. Let the vehicle's average staying time at each intersection be $\theta$, and let the probability of the emerging of $n$ different vehicles heading for $I_{j}$ at $I_{i}$ during $\theta$ be $P_{i, j}(n)$; therefore, the intersection connectivity $C_{i, j}$ for the two adjacent intersections $I_{i}$ and $I_{j}$ is defined as follows:

$$
C_{i, j}=\left\{\begin{array}{ll}
1 & \text { if } D_{i, j} \leq \delta \\
P_{i, j}(n \geq 1) & \text { otherwise }
\end{array} .\right.
$$

Suppose that $n$ different vehicles heading for $I_{j}$ emerge at $I_{i}$ by the Poisson distribution, which is defined as Eq. (2), the probability $P_{i, j}(n \geq 1)$ is determined by Eq. (3), as follows:

$$
\begin{aligned}
f\left(\lambda_{i, j} ; n\right)=\frac{\lambda_{i, j}{ }^{n} e^{-\lambda_{i, j}}}{n !} & , \text { where } \lambda_{i, j}=M_{i} * \alpha_{i, j} / \theta . \\
P_{i, j}(n \geq 1)= & 1-P_{i, j}(n=0) \\
= & 1-f\left(\lambda_{i, j} ; n=0\right)
\end{aligned}
$$




$$
=1-e^{-\lambda_{i, j}}
$$

Each intersection is usually connected to three-to-four different roadways, and has up to 12 moving directions at four-way intersections. The average traffic volumes passing through each intersection are different from each other. But, a tendency of the traffic flow between the intersections can be discovered by a statistical analysis of the traffic-volume records at each intersection. The terminology of "traffic influence" is used here to represent for the tendency of the traffic flow between intersections. Consequently, the probability that the emerging vehicles will move to a particular direction at each intersection can be determined by both the average traffic volume at each intersection and the traffic influence regarding the particular direction at the intersection. In Eq. (2), $M_{i}$ indicates the average traffic volume measured at $I_{i}$ during the time $\theta$, and $\alpha_{i, j}$ is the traffic influence from $I_{i}$ to $I_{j}$. The traffic influence can be obtained by a linear-regression analysis of the traffic amount heading to $I_{j}$ over the average traffic volume at $I_{i}$. For every single time interval $t$, let the total traffic amount moving from $I_{i}$ to $I_{j}$ during $t$ be $x_{i, j}^{t}$, and let the total traffic amount that passes through $I_{i}$ during $t$ be $y_{i}^{t} \cdot \alpha_{i, j}$ is calculated by Eq. (4), as follows:

$$
\alpha_{i, j}=\frac{k \sum_{t=1}^{k} x_{i, j}^{t} y_{i}^{t}-\sum_{t=1}^{k} x_{i, j}^{t} \sum_{t=i}^{k} y_{i}^{t}}{k \sum_{t=1}^{k}\left(x_{i, j}^{t}\right)^{2}-\left(\sum_{t=1}^{k} x_{i, j}^{t}\right)^{2}} .
$$

Without a loss of generality, the intersection connectivity can be extended to two arbitrary non-adjacent intersections. For two non-adjacent intersections that are denoted as $I_{i}$ and $I_{k}$, the message delivery from $I_{i}$ to $I_{k}$ should be done by vehicles passing through the intersections located on the path from $I_{i}$ to $I_{k}$; that is, for an intermediate intersection $I_{j}$ between $I_{i}$ and $I_{k}$, the traffic messages should be delivered to $I_{j}$ first, and then they can be delivered from $I_{j}$ to $I_{k}$. The $C_{i, k}$ is consequently defined as $C_{i, k}=C_{i, j} \times C_{j, k}$. Also, since there are many paths from $I_{i}$ to $I_{k}$ through other intersections, $C_{i, k}$ is defined as follows:

$$
C_{i, k}=\max \left\{\prod_{j=i}^{k} C_{j, j+1}\right\} .
$$

Here, the index $j+1$ indicates an adjacent intersection to $I_{j}$ on a path from $I_{i}$ to $I_{k}$. Consequently, the intersection connectivity for two arbitrary intersections is defined as follows:

$$
C_{i, k}=\left\{\begin{array}{lr}
1 & \text { if } D_{i, k} \leq \delta \\
P_{i, k}(n \geq 1) & \text { else if } I_{i} \text { and } I_{k} \text { are adjacent; } \\
\max \left\{\prod_{j=i}^{k} C_{j, j+1}\right\} & \text { otherwise }
\end{array}\right.
$$

The following table summarizes all notations used through our entire paper.

Table 1. Notations

\begin{tabular}{|c|l|}
\hline Notations & Descriptions \\
\hline$I$ & A set of all intersections \\
\hline$I_{i}$ & The $i$ th intersection in $I$ where $i=\{1, \ldots, n\}$ \\
\hline$P_{i, j}(n)$ & The probability of emerging $n$ vehicles moving from $I_{i}$ to $I_{j}$ \\
\hline$\alpha_{i, j}$ & The traffic influence from $I_{i}$ to $I_{j}$ \\
\hline$D_{i, j}$ & The distance from $I_{i}$ to $I_{j}$ \\
\hline$C_{i, j}$ & The intersection connectivity from $I_{i}$ to $I_{j}$ \\
\hline$\delta$ & The wireless transmission range \\
\hline$\theta$ & Vehicle's average staying time at an intersection \\
\hline$R S e t$ & A set of RSUs \\
\hline$A C_{k}$ & An AP-based cluster having $I_{k}$ as the head of the cluster. \\
\hline
\end{tabular}




\section{Intersection-Connectivity-Based RSU Allocation}

In this section, the authors' intersection-connectivity-based RSU-allocation strategy is described in detail. An AP algorithm is adopted to cluster the intersections. The AP algorithm is briefly reviewed first, and the RSU-allocation algorithms are then concretely provided in the subsections.

\subsection{Affinity Propagation (AP)}

AP clusters data points using the similarity between pairs of data points. The goal of AP is the finding of "exemplars," which is the center of each cluster. The similarity $s(i, k)$ indicates how well-suited the data point with index $k$ is to be the exemplar for data point $i$. A real preference value of each point is used for $s(k, k)$, and the points with larger values of $s(k, k)$ are more likely to be chosen as exemplars. Since AP considers all data points as potential exemplars, the $s(k, k)$ of each data point is set with the same preference. Based on this similarity, AP uses two kinds of the message that is exchanged between the data points. One is "responsibility" and the other is "availability." The responsibility $r(i, k)$, sent from the data point $i$ to the candidate exemplar point $k$, reflects the accumulated evidence for how well-suited point $k$ is to serve as the exemplar for point $i$, taking into account the other potential exemplars for point $i$. The availability $a(i, k)$, sent from the candidate exemplar point $k$ to the point $i$, reflects the accumulated evidence for how appropriate it would be for point $i$ to choose point $k$ as its exemplar, taking into account the support from other points that point $k$ should be an exemplar. $a(i, k)$ is initialized as zero: $a(i, k)=0$, and $r(i, k)$ and $a(i, k)$ are computed as follows:

$$
\begin{gathered}
r(i, k) \leftarrow s(i, k)-\max _{k^{\prime} s . t . k^{\prime} \neq k}\left\{a\left(i, k^{\prime}\right)+s\left(i, k^{\prime}\right)\right\} . \\
a(i, k) \leftarrow \min \left\{0, r(k, k)+\sum_{i^{\prime} \text { s.t. } i^{\prime} \notin\{i, k\}} \max \left\{0, r\left(i^{\prime}, k\right)\right\}\right\}, \\
a(k, k) \leftarrow \sum_{i^{\prime} \text { s.t.i } i^{\prime} \neq k} \max \left\{0, r\left(i^{\prime}, k\right)\right\} .
\end{gathered}
$$

For point $i$, the AP algorithm iteratively updates $r(i, k)$ and $a(i, k)$. And the point $k$ that maximizes $a(i, k)+r(i, k)$ becomes the exemplar for point $i$.

\subsection{AP-Based Connected-RSU Allocation}

Given a road network $G(I, R)$ such that $I$ is a set of intersections and $R$ is a set of roadways between two adjacent intersections, the intersection connectivity $C_{i, k}$ can be directly used to show how well the intersection $I_{k}$ is suited to be RSU candidate for the intersection $I_{i}$ because $C_{i, k}$ represents the message-delivery probability from $I_{i}$ to $I_{k}$. The proposed intersection connectivity is a probabilistic traffic model, so the similarity between a pair of intersections for the adoption of the AP algorithm is set to a loglikelihood of the intersection connectivity between the two intersections, as follows:

$$
s\left(I_{i}, I_{k}\right)=\log C_{i, k} \mid i \neq k .
$$

Since every intersection can be an RSU candidate, each intersection is supposed to have the same preference; therefore, for $i=k, s\left(I_{k}, I_{k}\right)$ is set to an average of all of the similarities, as follows:

$$
s\left(I_{k}, I_{k}\right)=\frac{\sum_{i=1}^{n} \sum_{j=1}^{n} s\left(I_{i}, I_{j}\right) \mid i \neq j}{n^{2}-n} .
$$


The previously mentioned AP algorithm clusters the intersections based on this similarity. The rule for the computing of the $r(i, k)$ and $a(i, k)$ is used. The AP-based CRSU-allocation algorithm is given below:

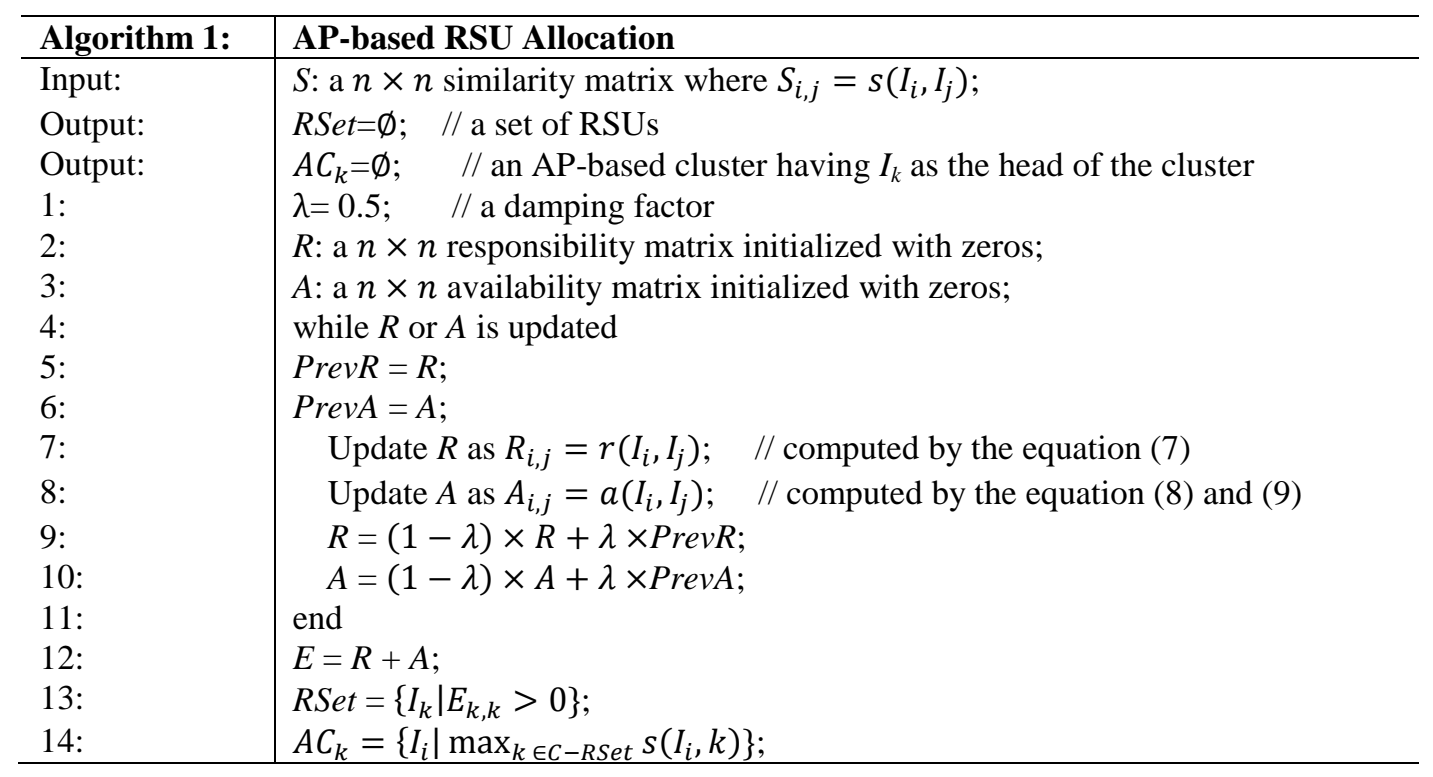

First of all, the RSU candidates for the maximizing of the traffic-message collection can be obtained by carrying out Algorithm 1, and these RSU candidates are denoted as "RSU-C." To obtain the RSU candidates for the maximizing of the traffic-message dissemination, Algorithm 1 can be performed with the transposed matrix of the above similarity matrix. Let these RSUs be "RSU-D." If it is assumed that the connected RSUs are installed so that the RSUs are connected through wire lines, the final RSU candidates are the union of RSU-C and RSU-D. The traffic messages collected by RSU-Cs can be instantly shared among other RSU-Cs and RSU-Ds, and then, the RSU-Ds can disseminate these messages back to the other intersections.

\subsection{AP-Based Wireless RSU Allocation}

In the previous section, the dedicated RSU candidates for message collection and message dissemination were selected separately. When those RSUs are connected to each other, it can provide the best performance for both message collection and dissemination. If only wireless RSUs can be installed, the above approach cannot provide the best solution. In this section, a wireless RSU-allocation strategy is therefore provided by modifying the similarity function.

The basic idea is the finding of common RSU positions that satisfy both message collection and dissemination. For a given pair of intersections denoted as $I_{i}$ and $I_{k}$, the similarity $s\left(I_{i}, I_{k}\right)$ from $I_{i}$ to $I_{k}$ is different to $s\left(I_{k}, I_{i}\right)$; however, to find the common RSU candidates for both message collection and dissemination, the similarity is modified so that both $s\left(I_{i}, I_{k}\right)$ and $s\left(I_{k}, I_{i}\right)$ share the same value. By choosing RSU positions based on the modified common similarity, the minimum number of RSUs required for covering all intersections can be also reduced. Here, two methodologies for assigning the common similarity between two intersections are suggested.

\section{(1) Average Similarity}

First, the average similarity can be simply considered. The similarity between $I_{i}$ and $I_{k}$ is set as the average value of both similarities, as follows:

$$
A V G_{i k}=\left(C_{i, k}+C_{k, i}\right) / 2 \text {, }
$$




$$
s\left(I_{i}, I_{k}\right)=s\left(I_{k}, I_{i}\right)=\log \left(A V G_{i k}\right) \quad \mid i \neq k .
$$

Since the average similarity reflects two connectivity values $C_{i, k}$ and $C_{k, i}$ impartially, the finding of RSU positions based on the average similarity can work reasonably in most bidirectional roadways where the two connectivity values are similar.

\section{(2) Weighted Similarity}

The second method considers the difference between $C_{i, k}$ and $C_{k, i}$. If the difference is small, the average value is similar to the original similarities; alternatively, if the difference is large, the average value can produce inaccurate results. For an example, suppose that the roadway between $I_{i}$ and $I_{k}$ is one-way, and $C_{i, k}$ is 0.9 but $C_{k, i}$ is 0 . Since $C_{i, k}$ is fairly large, $I_{k}$ could be a suitable position for message collection, but $I_{k}$ is not a recommendable position for message dissemination. If an average value is used, it is likely that $I_{k}$ is a suitable position for both message collection and message dissemination with at least a probability of 0.45 ; therefore, the proposed strategy considers the intersections with a small connectivity difference preferentially as RSU candidates, and the intersections with a large connectivity difference are excluded from the RSU candidates. To achieve this, the similarity is set as follows:

$$
\begin{aligned}
& \text { if }\left(\left|C_{i, k}-C_{k, i}\right|<\alpha\right) \text { then } \\
& \quad s\left(I_{i}, I_{k}\right)=s\left(I_{k}, I_{i}\right)=\log \left(\max \left(C_{i, k}, C_{k, i}\right)\right) \text {; } \\
& \text { else if }\left(\left|C_{i, k}-C_{k, i}\right|>(1-\alpha)\right) \text { then } \\
& \quad s\left(I_{i}, I_{k}\right)=s\left(I_{k}, I_{i}\right)=\log \left(\min \left(C_{i, k}, C_{k, i}\right)\right) ; \\
& \text { else } \quad s\left(I_{i}, I_{k}\right)=s\left(I_{k}, I_{i}\right)=\log \left(A V G_{i k}\right)
\end{aligned}
$$

Here, $\alpha$ indicates a threshold for the small connectivity difference such as $0<\alpha<0.5$. Using the modified similarities, common RSU candidates for both message collection and dissemination can be obtained by carrying out Algorithm 1 .

\section{Simulated Performance}

Now, the simulated performance of the proposed RSU-allocation model is analyzed. The results of the RSU allocation according to the following three different RSUallocation strategies are therefore compared: connected RSU allocation (C-RSU), wireless RSU allocation with the average similarity (W-RSU-AVG), and wireless RSU allocation with the weighted similarity (W-RSU-WG). The required minimum numbers of RSUs and positions, and the average coverage of the RSUs are mainly analyzed. The performance of the proposed model is also compared with that of the authors' previous Markov clustering-based approach (denoted as MC) and the K-means clustering results. For the simulation, the real traffic statistics and roadmaps of Seochogu, Korea, were used. The district has 89 intersections in total. Table 2 summarizes system parameters used in the simulation.

Table 2. Simulation Parameters

\begin{tabular}{|l|l|}
\hline \multicolumn{1}{|c|}{ Parameters } & \multicolumn{1}{c|}{ Values } \\
\hline Wireless-transmission range $(\delta)$ & $200 \mathrm{~m}, 250 \mathrm{~m}, 300 \mathrm{~m}$ \\
\hline Vehicle's average stay time at an intersection $(\theta)$ & $15 \mathrm{~s}$ \\
\hline Connectivity difference threshold $(\alpha)$ & $0.1,0.15,0.2$ \\
\hline
\end{tabular}




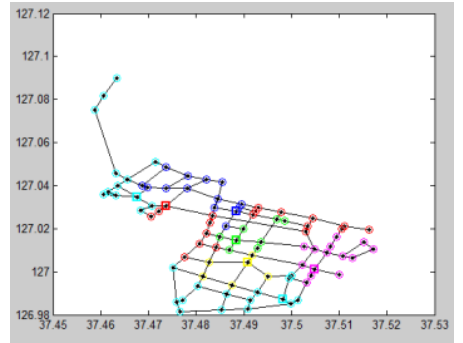

(1) C-RSU allocation for message collection and their coverage

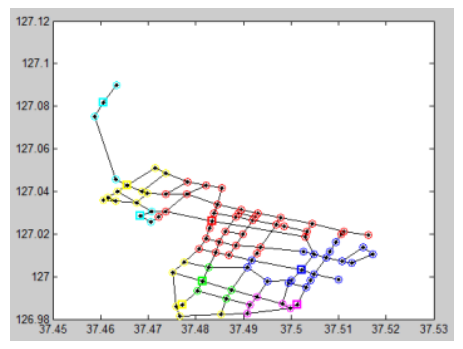

(4) W-RSU allocation with average similarity (W-RSU-AVG)

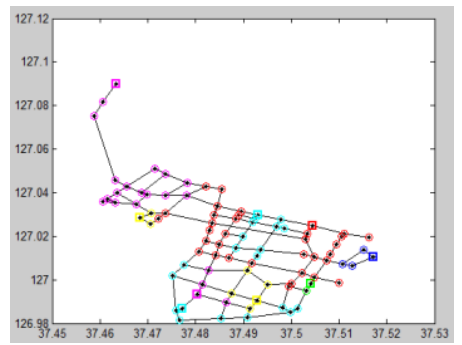

(2) C-RSU allocation for message dissemination

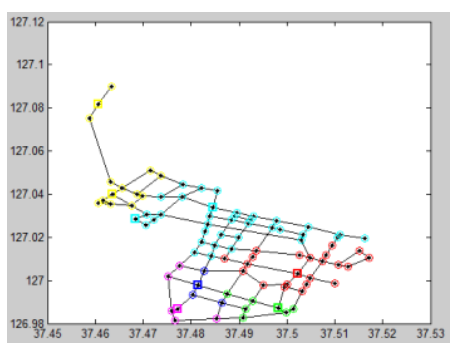

(5) W-RSU allocation with weighted similarity $(\alpha=0.1)$ (W-RSU-WG-0.1)

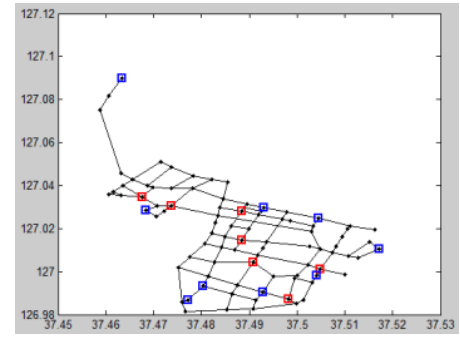

(3) Positions of collection RSUs (marked as red square) and dissemination RSUs (marked as blue square)

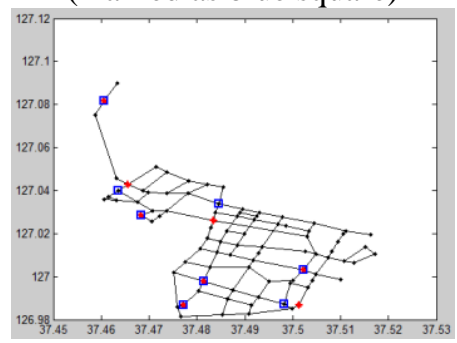

(6) Positions of W-RSU-AVG (marked as red star) and W-RSU-WG-0.1 (marked as blue square)

Figure 1. The Results of the RSU Allocation According to the Three Different Strategies $(\delta=250 \mathrm{~m}, \alpha=0.1)$

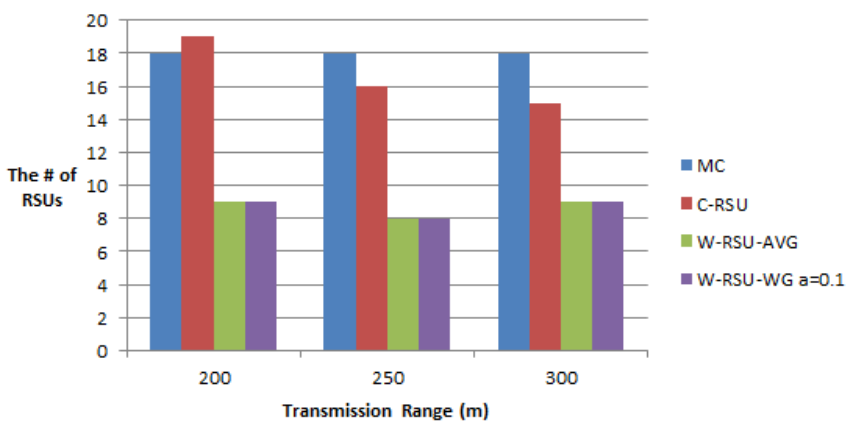

Figure 2. The Number of RSUs According to Different RSU Allocation Strategies

Figure 1 shows the positions of the RSUs and their coverage according to the three RSU-allocation strategies. Figure 2 summarizes the minimum number of RSUs to cover all intersections according to the proposed three strategies and the Markov clusteringbased strategy (MC). As shown in the Figure 2, the proposed wireless RSU-allocation model requires a lesser number of RSUs. The MC allocation and C-RSU allocation require more than 15 numbers of RSUs, but W-RSU-AVG and W-RSU-WG require less than 10 numbers of RSUs. 


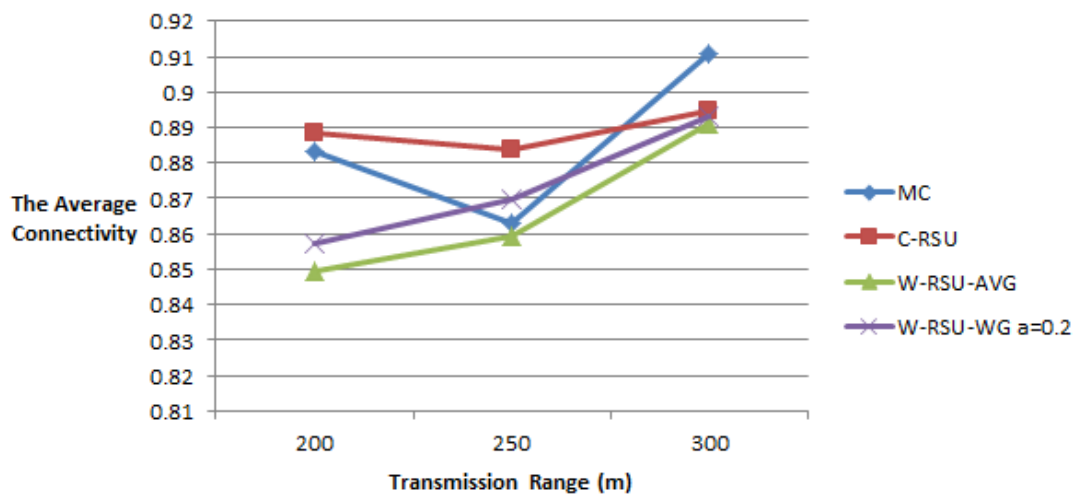

\section{Figure 3. The Average Coverage of RSUs according To Different RSU Allocation Strategies}

Figure 3 shows the average probability for both traffic data collection and dissemination (denoted as "average connectivity") according to diverse RSU allocation strategies. As shown in the figure, the MC allocation and the C-RSU allocation shows the best average connectivity. The RSUs in the MC strategies can communicate with intersections covered by them with an average probability of about 0.88 when the transmission range is $200 \mathrm{~m}$. The average connectivity of RSUs in the C-RSU strategy is about 0.89 . On the other hands, the wireless RSUs shows a lesser performance than the connected RSUs in the average connectivity. It is natural because wireless RSU allocation strategies find common RSUs (not dedicated RSUs chosen separately for the data collection and dissemination) by considering both data collection and dissemination. In the case where the average similarity is considered, the average connectivity of W-RSUAVG is about 0.85 . When the weighted similarity with a weight threshold of 0.2 is considered, the average connectivity of W-RSU-WG is about 0.86 . As shown in the figure, the W-RSU-WG shows the middle level of performance between the C-RSU allocation and the W-RSU-AVG allocation. The wireless RSU allocation strategies produce a little lesser performance in the sense of the average connectivity but they reduced the number of RSUs significantly. In addition, W-RSUs do not need to be connected through wired lines. Thus, we can conclude that the proposed wireless RSU allocation strategies find practical and reasonable RSU positions for satisfying with both connectivity and economics.

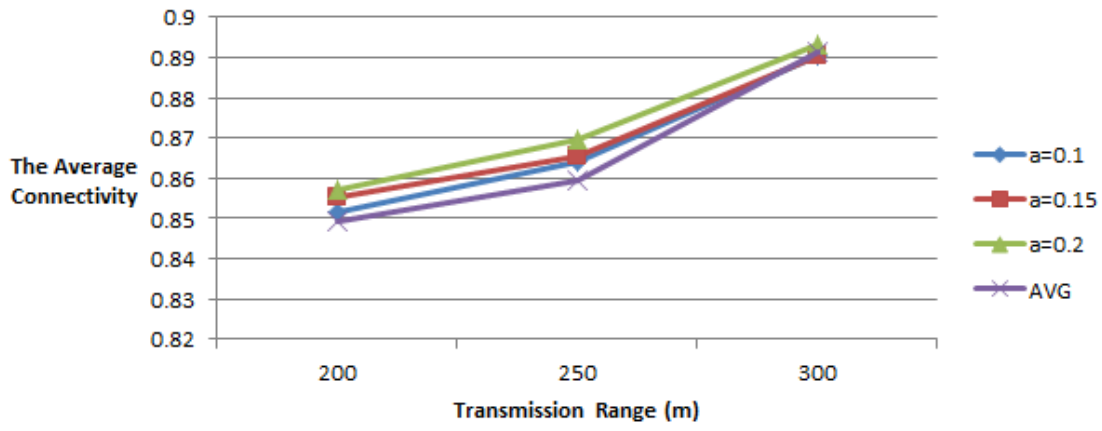

Figure 4. The Average Connectivity of RSUs According To Weight Threshold

$\alpha$

Figure 4 shows the average connectivity of W-RSU-WG according to the weight threshold $\alpha$. The W-RSU-WG shows slightly better performance than the W-RSU-AVG in the sense of average connectivity. And the W-RSU-WG with a weight threshold of 0.2 showed the best performance in Seochogu district. But the results will be dependent on 
the roadway situation. If a district has a road map that consists of lots of one-way roadways, then W-RSU-WG can work efficiently. But in most roadways where the bidirectional connectivity does not differ so much, W-RSU-AVG and W-RSU-WG will show the similar performance.

The performance of the proposed model was also compared with the existing clustering-based approaches. Table 4 summarizes the comparison results. When compared with the K-means clustering for the installation of 10 RSUs, the proposed model still shows a superior performance for the average connectivity. Also, the performance of the $\mathrm{K}$-means clustering is deeply affected by the initial cluster-center positions that are randomly chosen, so the results do not show a constant performance all the time. The Kmeans results given in the table show the best performances that were obtained from repeated simulations. From the simulated analysis with the real-field traffic data, it is possible to conclude that the proposed RSU-allocation model provides the best solutions for the maximization of the RSU coverage for intersections.

Table 3. Comparison with K-means Clustering

\begin{tabular}{|c|c|c|}
\hline & $\begin{array}{c}\text { K-means with AVG } \\
\text { similarity }\end{array}$ & $\begin{array}{c}\text { K-means with WG } \\
\text { similarity }(\boldsymbol{\alpha}=\mathbf{0 . 1})\end{array}$ \\
\hline The minimum \# of RSUs & 10 & 10 \\
\hline The average connectivity & 0.81 & 0.825 \\
\hline
\end{tabular}

$(\delta=250 \mathrm{~m})$

\section{Conclusion}

In this paper, a concept of intersection connectivity that represents the messagedelivery probability between two arbitrary intersections has finally been formalized. By using a probabilistic traffic-delivery model, novel RSU-allocation strategies that can maximize the RSU coverage for intersections by considering both message collection and message dissemination are proposed. By exploiting an AP algorithm to make clusters of intersections, three types of RSU-allocation strategy have been proposed, and optimal RSU positions that can maximize both the average message-collection probability and the average message-dissemination probability have been obtained as a result. The RSUs that were selected using the proposed model provide a more-effective performance in terms of both message collection and dissemination compared with those of the existing approaches.

\section{Acknowledgment}

This research was supported by the Basic Science Research Program through the National Research Foundation of Korea (NRF), funded by the Ministry of Education, Science and Technology (No.NRF-2014R1A1A3053491).

\section{References}

[1] K. Barrachina, P. Garrido, J. Cano, C. T. Calafate, Road Side Unit Deployment: A Density-Based Approach, IEEE Intelligent Transportation System Magazine, (2013), pp.30-39.

[2] J. Chi, Y. Jo, H. Park, T. Hwang and S. Park, "An Effective RSU Allocation Strategy for Maximizing Vehicular Network Connectivity", International Journal of Control and Automation, vol. 6, no.4, (2013).

[3] J. Chi, H. Park, T. Hwang and S. Park, "An Extended Intersection-Priority based RSU Allocation for VANET”, Information Journal, pp.1419-1432, vol.17, no.4, (2014).

[4] J. Chi, S. Do, Y. Kim and S. Park, "Deployment of Roadside Units based on Markov Clustering Algorithm", International Journal of Applied EngineeringResearch, (2015), pp.33558-33561.

[5] J. Chi, S. Do and S. Park, "Traffic Flow-based Roadside Unit Allocation Strategy for VANET", Proc. of International Conference on Big Data and Smart Computing (BigComp), (2016).

[6] B. J. Frey and D. Dueck, "Clustering by Passing Messages Between Data Points", Science, vol. 315, (2007). 
[7] C. M. Silva, A.L.L. Auino and W. Meira, "Deployment of roadside units based on partial mobility information", Computer Communications, vol.60, (2015), pp.28-39.

[8] P. Patil and A. Gokhale, "Voronoi-based Placement of Road-side Units to Improve Dynamic Resource Management in Vehicluar Ad Hoc Networks", Proc. Of the IEEE Collaboration Technologies and Systems (CTS), (2013).

[9] M. Rios, V. Marianov and M. Perez, "Locating fixed roadside units in a bus transport network for maximum communications probability", Transportation Research Part C, (2015), pp.35-47.

[10] C. Wang, X. Li, F. Li and H. Lu, "A Mobility Clustering-based Roadside Units Deployment for VANET”, Proc. Of Asia-Pacific Network Operation and Management Symposium (APNOMS), (2014).

\section{Authors}

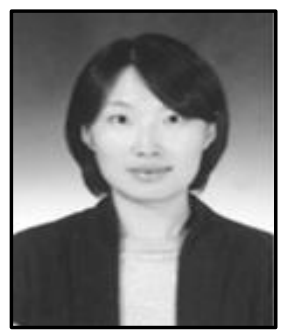

\section{Soyoung Park}

2006. 2, Ph.D at Ewha Womans University, Korea 2006. 1. 2010. 1, Post-doctoral Researcher,

University of Central Florida, U.S.A

2010. 3 2012. 2, Full-time Lecturer, Ewha Womans University, Korea 2012. 3 2014. 2, Full-time Lecturer, Konkuk University, Korea 2014. 3 Present, Assistant Professor, Konkuk University, Korea Research Interests: Cryptography, Network security, Vehicular network

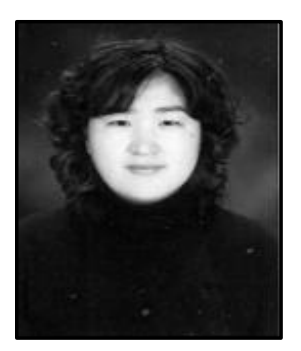

\section{Jeonghee Chi}

2006. 2, Ph.D at Chungbuk National University, Korea 2007. 3 2015. 2, Full-time Lecturer, Konkuk University, Korea 2015. 3 Present, Assistant Professor, Konkuk University, Korea Research Interests: Data management in mobile ad-hoc networks, Location-based information systems,

Vehicle ad hoc network 
International Journal of Control and Automation

Vol. 10, No. 1 (2017) 